\title{
The success rate and precision of GPS ambiguities
}

\author{
P. J. G. Teunissen \\ Department of Mathematical Geodesy and Positioning, Faculty of Civil Engineering and Geosciences, \\ Delft University of Technology, Thijsseweg 11, 2629 JA Delft, The Netherlands \\ e-mail: p.j.g.teunissen@geo.tudelft.nl; Tel.: + 3115278 2558; Fax: + 31152783711
}

Received: 11 January 1999 / Accepted: 2 November 1999

\begin{abstract}
An application of a theorem on the optimality of integer least-squares (LS) is described. This theorem states that the integer LS estimator maximizes the ambiguity success rate within the class of admissible integer estimators. This theorem is used to show how the probability of correct integer estimation depends on changes in the second moment of the ambiguity 'float' solution. The distribution of the 'float' solution is considered to be a member of the broad family of elliptically contoured distributions. Eigenvalue-based bounds for the ambiguity success rate are obtained.
\end{abstract}

Key words: GPS - Integer LS - Ambiguity precision Ambiguity success rate

\section{Introduction}

Global positioning system (GPS) models on which ambiguity resolution is based can all be cast in the following conceptual frame of linear(ized) observation equations:

$y=A a+B b+e$

where $y$ is the given GPS data vector of order $m, a$ and $b$ are the unknown parameter vectors respectively of order $n$ and $o$, and $e$ is the noise vector. The matrices $A$ and $B$ are the corresponding design matrices. The data vector $y$ will usually consist of the 'observed minus computed' single- or dual-frequency double-difference (DD) phase and/or pseudorange (code) observations accumulated over all observation epochs. The entries of vector $a$ are then the DD carrier-phase ambiguities, expressed in units of cycles rather than range. They are known to be integers, $a \in Z^{n}$. The entries of the vector $b$ will consist of the remaining unknown parameters, such as, for instance, baseline components (coordinates) and possibly atmospheric delay parameters (troposphere, ionosphere). They are known to be real valued, $b \in R^{o}$.
The structure of the above conceptual model applies to a great variety of GPS models currently in use. They range from single-baseline models used for kinematic positioning to multi-baseline models used as a tool for studying geodynamic phenomena. An overview of these and other GPS models, together with their application in surveying, navigation and geodesy, can be found in textbooks such as those of Leick (1995), Parkinson and Spilker (1996), Hofmann-Wellenhof et al. (1997), Strang and Borre (1997) and Teunissen and Kleusberg (1998).

The procedure which is usually followed for solving the GPS model of Eq. (1) can be divided into three steps (for more details we refer to e.g. Teunissen 1993 or de Jonge and Tiberius 1996). In the first step we simply disregard the integer constraints $a \in Z^{n}$ on the ambiguities and perform a standard adjustment. As a result we obtain the (real-valued) estimates of $a$ and $b$, together with their variance-covariance (VC) matrix

$\left[\begin{array}{l}\hat{a} \\ \hat{b}\end{array}\right], \quad\left[\begin{array}{cc}Q_{\hat{a}} & Q_{\hat{a} \hat{b}} \\ Q_{\hat{b} \hat{a}} & Q_{\hat{b}}\end{array}\right]$

This solution is referred to as the 'float' solution. In the second step the 'float' ambiguity estimate $\hat{a}$ is used to compute the corresponding integer ambiguity estimate $\check{a}$. This implies that a mapping $F: R^{n} \mapsto Z^{n}$, from the $n$-dimensional space of reals to the $n$-dimensional space of integers, is introduced such that

$\check{a}=F(\hat{a})$

Once the integer ambiguities are computed, they are used in the third step to finally correct the 'float' estimate of $b$. As a result we obtain the 'fixed' solution $\check{b}=\hat{b}-Q_{\hat{b} \hat{a}} Q_{\hat{a}}^{-1}(\hat{a}-\check{a})$.

In this contribution we will study some aspects of the probabilistic consequences of Eq. (3). In particular, we study the dependence of the probability of correct integer estimation on the VC matrix of the ambiguity 'float' solution. For that purpose, we first give a brief review of the theory of integer ambiguity estimation in Sect. 2. Special attention is given to the optimality property of 
the integer LS estimator. The theorem presented gives a probabilistic justification for using the integer LS estimator. The probability of correct integer ambiguity estimation will also be referred to as the ambiguity success rate, being the frequentist interpretation of this probability. In the two sections following, the theorem is used to prove a number of results regarding the relation between the ambiguity success rate and ambiguity precision.

\section{Optimality of integer LS}

There are many ways of computing an integer ambiguity vector $\check{a}$ from its real-valued counterpart $\hat{a}$. To each such method belongs a mapping $F: R^{n} \mapsto Z^{n}$ from the $n$-dimensional space of real numbers to the $n$-dimensional space of integers. Due to the discrete nature of $Z^{n}$, the map $F$ will not be one-to-one, but instead a many-toone map. This implies that different real-valued ambiguity vectors will be mapped to the same integer vector. We can therefore assign a subset $S_{z} \subset R^{n}$ to each integer vector $z \in Z^{n}$

$S_{z}=\left\{x \in R^{n} \mid z=F(x)\right\}, \quad z \in Z^{n}$

The subset $S_{z}$ contains all real-valued ambiguity vectors that will be mapped by $F$ to the same integer vector $z \in Z^{n}$. This subset is referred to as the pull-in region of $z$ (Jonkman 1997; Teunissen 1998b). It is the region in which all ambiguity 'float' solutions are pulled to the same 'fixed' ambiguity vector $z$. Using the pull-in regions, we can give an explicit expression for the corresponding integer ambiguity estimator. It reads

$\check{a}=\sum_{z \in Z^{n}} z s_{z}(\hat{a})$

with the indicator function

$s_{z}(\hat{a})= \begin{cases}1 & \text { if } \hat{a} \in S_{z} \\ 0 & \text { otherwise }\end{cases}$

Since the pull-in regions define the integer estimator completely, we can define classes of integer estimators by imposing various conditions on the pull-in regions. In Teunissen (1998c) we defined one such class, which we called the class of admissible integer estimators. These integer estimators are defined as follows.

Definition. The integer estimator $\check{a}=\sum_{z \in Z^{n}} z s_{z}(\hat{a})$ is said to be admissible if

1. $\bigcup_{z \in Z^{n}} S_{z}=R^{n}$

2. $S_{z_{1}} \cap S_{z_{2}}=\emptyset, \quad \forall z_{1}, z_{2} \in Z^{n}, \quad z_{1} \neq z_{2}$

3. $S_{z}=z+S_{0}, \quad \forall z \in Z^{n}$

This definition was motivated as follows. Each one of the above three conditions describes a property of which it seems reasonable that it is possessed by an arbitrary integer ambiguity estimator. The first condition states that the pull-in regions should not leave any gaps and the second that they should not overlap. The absence of gaps is needed in order to be able to map any 'float' solution $\hat{a} \in R^{n}$ to $Z^{n}$, while the absence of overlaps is needed to guarantee that the 'float' solution is mapped to just one integer vector. Note that we allow the pull-in regions to have common boundaries. This is permitted if we assume to have zero probability that $\hat{a}$ lies on one of the boundaries. This will be the case when the probability density function (pdf) of $\hat{a}$ is continuous.

The third and last condition follows from the requirement that $F(x+z)=F(x)+z, \forall x \in R^{n}, z \in Z^{n}$. This condition is a reasonable one to ask for. It states that when the 'float' solution is perturbed by $z \in Z^{n}$, the corresponding integer solution is perturbed by the same amount. This property allows us to apply the integer remove-restore technique: $F(\hat{a}-z)+z=F(\hat{a})$. It therefore allows us to work with the fractional parts of the entries of $\hat{a}$, instead of with its complete entries.

Although various integer estimators exist which are admissible, some may be better than others. Having the problem of GPS ambiguity resolution in mind, we are particularly interested in the estimator which maximizes the ambiguity success rate. For that purpose we first need the probability mass function (pmf) of the integer estimator. Since

$\check{a}=z \quad \Leftrightarrow \quad \hat{a} \in S_{z}$

we have $P(\check{a}=z)=P\left(\hat{a} \in S_{z}\right)$. The pmf of $\check{a}$ follows therefore as

$P(\check{a}=z)=\int_{S_{z}} p_{a}(x) \mathrm{d} x, \quad \forall z \in Z^{n}$

where $p_{a}(x)$ denotes the pdf of the 'float' solution $\hat{a}$. The subscript $a$ is used to show that the pdf still depends on the unknown ambiguity vector $a \in Z^{n}$.

From Eq. (7) the probability of correct integer estimation follows as $P(\check{a}=a)$. This probability will be different for different estimators. In order to find the estimator which gives the largest ambiguity success rate, we need to know which estimator maximizes $P(\check{a}=a)$. The answer to this question is given by the following theorem.

Theorem 1. (Teunissen) Let the integer LS estimator be defined as

$\check{a}_{\mathrm{LSQ}}=\arg \min _{z \in Z^{n}}\|\hat{a}-z\|_{Q_{\hat{a}}}^{2}$

and the pdf of $\hat{a}$ be given as

$p_{a}(x)=\sqrt{\operatorname{det}\left(Q_{\hat{a}}^{-1}\right)} G\left(\|x-a\|_{Q_{\hat{a}}}^{2}\right)$

where $G: R \mapsto[0, \infty)$ is decreasing and $Q_{\hat{a}}$ is positive definite. Then

$P\left(\check{a}_{\mathrm{LSQ}}=a\right) \geq P(\check{a}=a)$

for any admissible estimator $\check{a}$. 
This theorem gives a probabilistic justification for using the integer LS estimator. For GPS ambiguity resolution it shows that we are better off using the integer LS estimator than any other admissible integer estimator. The theorem was introduced and proved in Teunissen (1998c). The family of distributions defined in Eq. (9) is known as the family of elliptically contoured distributions (Chmielewsky 1981). Several important distributions belong to this family. The multivariate distribution can be shown to be a member of this family by choosing $G(x)=(2 \pi)^{-\frac{n}{2}} \exp -\frac{1}{2} x, x \in R$. Another member is the multivariate $t$ distribution.

In the sections following, we will use the above theorem to prove a number of conjectures concerning the relation between the ambiguity success rate and ambiguity precision. Some of these results also allow us to compute bounds for the ambiguity success rate.

\section{The effect of using an approximate ambiguity VC matrix}

It is well known from standard LS theory that the linear unbiased (real-valued) LS estimator may lose its optimality property of minimum variance when a positive-definite matrix other than the $\mathrm{VC}$ matrix is used for the weighting of the data. We may expect that a similar situation will hold true for the integer LS estimator. That is, we expect the integer LS estimator to lose its optimality property of maximizing the probability of correct integer estimation when an arbitrary positive-definite matrix is used for the weighting. That this is true can be shown as a direct consequence of the above theorem.

Corollary 1. Let $\Sigma$ be any positive-definite matrix of order $n$ and define

$\check{a}_{\Sigma}=\arg \min _{z \in Z^{n}}\|\hat{a}-z\|_{\Sigma}^{2}$

Then $\check{a}_{\Sigma}$ is admissible and

$P\left(\check{a}_{\mathrm{LSQ}}=a\right) \geq P\left(\check{a}_{\Sigma}=a\right)$

Proof. In order to prove this corollary, we only need to show that $\check{a}_{\Sigma}$ is admissible. Once this has been established, the stated result of Eq. (12) follows from theorem 1. The admissibility can be shown as follows. The first two conditions of the definition are satisfied, since Eq. (11) produces - apart from boundary ties - a unique integer vector for any 'float' solution $\hat{a} \in R^{n}$. And since $\check{a}_{\Sigma}=\arg \min _{z \in Z^{n}}\|\hat{a}-u-z\|_{\Sigma}^{2}+u$ holds true for any integer $u \in Z^{n}$, the integer remove-restore technique also applies.

As the corollary shows, a proper choice of the data weight matrix is also of importance for ambiguity resolution. The choice of weights is optimal when the weight matrix equals the inverse of the ambiguity VC matrix. A too optimistic precision description or a too pessimistic precision description will both result in a less than optimal ambiguity success rate. In the case of GPS, the observation equations (the functional model) are sufficiently known and well documented. However, the same cannot be said of the VC matrix of the GPS data. In the many GPS textbooks available, we will usually find only a few comments, if any, on this VC matrix. Only a few studies have been reported in the literature. Examples are as follows: Euler and Goad (1991), Gerdan (1995), Gianniou (1996), and Jin and de Jong (1996), who studied the elevation dependence of the observation variances; Jonkman (1997) and Tiberius (1998), who considered time correlation and cross correlation of the pseudo ranges and carrier phases as well; and Schaffrin and Bock (1988), Bock (1998) and Teunissen (1998a), who considered the inclusion of stochastic ionospheric constraints.

A systematic study of the stochastic model is of course far from trivial. Not only do the noise characteristics depend on the mechanization of the measurement process, and therefore on the make and type of the receiver used, but the residual terms which are not captured by the observation equations, such as environmental effects, will also have their influence. Despite these difficulties though, we believe that the time has come to put more research effort into the stochastic model. Examples showing that improved stochastic modelling indeed pays off in terms of an increased ambiguity success rate can be found in Jonkman (1997) and Teunissen et al. (1998).

Quite another aspect made clear by the corollary, is the relation between 'integer rounding' and 'integer LS'. One of the simplest choices for $\Sigma$ would be a diagonal matrix. In that case $\|\hat{a}-z\|_{\Sigma}^{2}$ reduces to a sum of squares and $\check{a}_{\Sigma}$ becomes the integer estimator that follows from a rounding to the nearest integer of the entries of $\hat{a}$. Thus $\check{a}_{\Sigma}=[\hat{a}]$, where ' $[\cdot]$ ' denotes the operation of componentwise rounding, and

$P\left(\check{a}_{\mathrm{LSQ}}=a\right) \geq P([\hat{a}]=a)$

We can generalize this result to a whole class of integer estimators based on rounding, when the choice $\Sigma=\left(Z^{T} D Z\right)^{-1}$ is made, where $D$ is a diagonal matrix with positive entries and $Z$ is an admissible ambiguity transformation. Ambiguity transformations are said to be admissible when all the entries of both $Z$ and its inverse are integer (Teunissen 1995). For this particular choice of $\Sigma$, we have $\|\hat{a}-z\|_{\Sigma}^{2}=(Z \hat{a}-u)^{T} D(Z \hat{a}-u)$, with $u=Z z \in Z^{n}$. Hence, when parametrized in $u$, $\|\hat{a}-z\|_{\Sigma}^{2}$ again reduces to a sum of squares. Thus $\check{u}=[Z \hat{a}]$ and $\check{a}_{\Sigma}=Z^{-1}[Z \hat{a}]$. In this case the integer estimator is computed by first transforming the 'float' solution, then applying the componentwise rounding scheme, followed by the back transformation. For the probability of correct integer estimation, we thus have

$P\left(\check{a}_{\mathrm{LSQ}}=a\right) \geq P\left(Z^{-1}[Z \hat{a}]=a\right)$

for any admissible ambiguity transformation $Z$.

Note that Eq. (13) is a special case of Eq. (14). The choice $Z=I_{n}$, however, is not usually the best one. That 
is, we can often improve upon the ambiguity success rate by choosing an appropriate transformation matrix $Z$. This is particularly true in the case of GPS, when the DD ambiguities are used. Since the equality in Eq. (14) will hold true in the case that the VC matrix of $Z \hat{a}$ is diagonal, an ambiguity transformation should be used that results in an as close to diagonal form as possible. This is achieved when using the decorrelation process of the LAMBDA method. Hence, when we decide to use the integer estimator based on rounding, we should at least decorrelate the ambiguities first, before applying the integer rounding scheme. In this way we will obtain a success rate which is higher than the one obtained without using the decorrelation process.

\section{The effect of improving the ambiguity precision}

Another result which we may conjecture on intuitive grounds is the increase in the success rate when the ambiguity precision improves. This is not too difficult to prove when the distribution of the 'float' solution is normal and the ambiguity VC matrix is diagonal. In that case we can make direct use of the independence between the individual ambiguities. We will show, however, that the result also holds true when the distribution is elliptically contoured and the VC matrix is nondiagonal.

Before stating the theorem, we first specify the notion of 'precision improvement' in the case that the VC matrix is nondiagonal. Let $\Sigma_{1}$ and $\Sigma_{2}$ be the VC matrices of the two 'float' solutions $\hat{a}^{\Sigma_{1}}$ and $\hat{a}^{\Sigma_{2}}$. The precision of the first estimator is then said to be better or as good as the precision of the second estimator, when the variance of every linear function of $\hat{a}^{\Sigma_{1}}$ is smaller than or as large as the variance of the same function of $\hat{a}^{\Sigma_{2}}$. Thus $f^{T} \Sigma_{2} f \geq f^{T} \Sigma_{1} f$ must hold true for every $f \in R^{n}$. The difference of these two VC matrices must therefore be positive semidefinite, or, $\Sigma_{2} \geq \Sigma_{1}$.

Theorem 2. Let the integer LS estimator be given as

$\check{a}_{\mathrm{LSQ}}^{\Sigma}=\arg \min _{z \in Z^{n}}\left\|\hat{a}^{\Sigma}-z\right\|_{\Sigma}^{2}$

and the pdf of $\hat{a}^{\Sigma}$ as

$p_{a}^{\Sigma}(x)=\sqrt{\operatorname{det}\left(\Sigma^{-1}\right)} G\left(\|x-a\|_{\Sigma}^{2}\right)$

where $G: R \mapsto[0, \infty)$ is decreasing and $\Sigma$ is positive definite. If $\Sigma_{2} \geq \Sigma_{1}$, then

$P\left(\check{a}_{\mathrm{LSQ}}^{\Sigma_{1}}=a\right) \geq P\left(\check{a}_{\mathrm{LSQ}}^{\Sigma_{2}}=a\right)$

Proof. The proof follows from combining our theorem 1 with a theorem of Anderson (1996). According to Anderson's theorem, if $\Sigma_{2} \geq \Sigma_{1}$, then $P\left(\hat{a}^{\Sigma_{1}} \in C_{a}\right) \geq$ $P\left(\hat{a}^{\Sigma_{2}} \in C_{a}\right)$, for any convex set $C_{a} \subset R^{n}$ symmetric about $a$. Since the pull-in region of the integer LS estimator $\check{a}_{\mathrm{LSQ}}^{\Sigma_{2}}, S_{a}^{\Sigma_{2}}=\left\{x \in R^{n} \mid a=\arg \min _{z \in Z^{n}}\|x-z\|_{\Sigma_{2}}^{2}\right\}$, is convex and symmetric about $a$, we may take $C_{a}=S_{a}^{\Sigma_{2}}$, which gives us the inequality

1. $P\left(\hat{a}^{\Sigma_{1}} \in S_{a}^{\Sigma_{2}}\right) \geq P\left(\hat{a}^{\Sigma_{2}} \in S_{a}^{\Sigma_{2}}\right)=P\left(\check{a}_{\mathrm{LSQ}}^{\Sigma_{2}}=a\right)$

According to our theorem $1, P\left(\check{a}_{\mathrm{LSQ}}^{\Sigma_{1}}=a\right) \geq P(\check{a}=a)$ for any admissible estimator $\check{a}$. If this admissible estimator is chosen to be the one that uses the pull-in regions of $\check{a}_{\mathrm{LSQ}}^{\Sigma_{2}}$ to map $\hat{a}^{\Sigma_{1}}$, we have $P(\check{a}=a)=$ $P\left(\hat{a}^{\Sigma_{1}} \in S_{a}^{\Sigma_{2}}\right)$, and therefore

2. $P\left(\check{a}_{\mathrm{LSQ}}^{\Sigma_{1}}=a\right) \geq P\left(\hat{a}^{\Sigma_{1}} \in S_{a}^{\Sigma_{2}}\right)$

Combining (1) with (2) proves the theorem.

In words the theorem states that the ambiguity success rate gets larger when the precision improves. We remark that the theorem should not be confused with corollary 1 . In corollary 1 , the two probabilities of the inequality of Eq. (12) are evaluated with respect to the same pdf, $p_{a}(x)$. Moreover, no conditions are imposed on the definiteness of the difference $Q_{\hat{a}}-\Sigma$. That is, either $Q_{\hat{a}} \geq \Sigma$ or $\Sigma \geq Q_{\hat{a}}$ may hold true. In theorem 2, the two probabilities of Eq. (17) are evaluated with respect to two different pdfs, namely $p_{a}^{\Sigma_{1}}(x)$ and $p_{a}^{\Sigma_{2}}(x)$. And, in addition, the condition $\Sigma_{2} \geq \Sigma_{1}$ is imposed.

As an almost direct consequence of theorem 2, we have the following corollary.

Corollary 2. Let $y \in R^{m}$ be distributed as $y \sim N(A a+B b$, $Q_{y}$ ) with $a \in Z^{n}, b \in R^{o}$ and the constraints $C^{T} b=c \in R^{p}$. Then

$P\left(\check{a}_{\mathrm{LSQ}}^{C}=a\right) \geq P\left(\check{a}_{\mathrm{LSQ}}=a\right)$

where the constraints $C^{T} b=c$ were used for computing the integer LS estimator $\check{a}_{\mathrm{LSQ}}^{C}$ and not used in the computation of $\check{a}_{\mathrm{LSQ}}$.

Proof. When disregarding the integer constraints $a \in Z^{n}$, the two real-valued LS estimators of $a$, based on imposing, respectively not imposing, the constraints $C^{T} b=c$, are distributed as $\hat{a}^{C} \sim N\left(a, Q_{\hat{a}^{c}}\right)$ and $\hat{a} \sim N\left(a, Q_{\hat{a}}\right)$. Since the two $\mathrm{VC}$ matrices are related as

$Q_{\hat{a}^{c}}=Q_{\hat{a}}-Q_{\hat{a} \hat{b}} C\left(C^{T} Q_{\hat{b}} C\right)^{-1} C^{T} Q_{\hat{b} \hat{a}}$

we have $Q_{\hat{a}^{c}} \leq Q_{\hat{a}}$. The corollary now follows from theorem 2.

This result shows that we may improve upon the probability of correct integer estimation by including constraints on the real-valued parameter vector $b \in R^{o}$. In the context of GPS ambiguity resolution, the vector $b$ may consist of DD receiver-satellite ranges, baseline components and/or atmospheric (ionosphere, troposphere) delays.

In the case of GPS, there are various applications where we can see this corollary at work. Three such examples are given. The ambiguity success rate of the geometry-free GPS model is usually experienced to be poorer than that of the geometry-based GPS model. The reason lies in the different parameterizations used 
for the two models. In the case of the geometry-free model, the observation equations are parametrized in terms of the DD ranges. Hence, the relative receiversatellite geometry which ties these $\mathrm{DD}$ ranges to a common baseline is not used in this model. These constraints are however used in the geometry-based model.

As a second example consider the influence of the ionosphere. Ambiguity resolution is known to be particularly successful in the case of short baselines. For sufficiently short baselines, we can often constrain the ionospheric delays to be zero. Since this is not permitted for longer baselines, more parameters will be included in the model and thus less constraints will be imposed. Finally, consider the problem of GPS attitude determination. Ambiguity resolution is also often successful in this case. The reason lies in the assumed absence of the atmospheric delays and the additional geometric constraints that may be imposed on the configuration of the baselines.

As a completely different application, theorem 2 can also be used to formulate easy-to-compute bounds for the success rate. This is particularly useful in case the success rate itself is difficult to compute. A large enough lower bound would then inform us whether ambiguity resolution can expected to be successful, while a too small upper bound would indicate that successful ambiguity resolution cannot be expected. Two such bounds are given in the following corollary.

Corollary 3. Let $\hat{a} \in R^{n}$ be distributed as $\hat{a} \sim N\left(a, Q_{\hat{a}}\right)$ and let $\check{a}_{\mathrm{LSQ}}$ be the integer LS estimator of $a \in Z^{n}$. Then

$$
\begin{aligned}
\left(2 \Phi\left(\frac{1}{2 \sqrt{\lambda_{\max }}}\right)-1\right)^{n} & \leq P\left(\check{a}_{\mathrm{LSQ}}=a\right) \\
& \leq\left(2 \Phi\left(\frac{1}{2 \sqrt{\lambda_{\min }}}\right)-1\right)^{n}
\end{aligned}
$$

with $\lambda_{\min }, \lambda_{\max }$ the extreme eigenvalues of $Q_{\hat{a}}$ and

$\Phi(x)=\int_{-\infty}^{x} \frac{1}{\sqrt{2 \pi}} \exp \left\{-\frac{1}{2} y^{2}\right\} \mathrm{d} y$

Proof. In order to prove the two bounds, we have to apply theorem 2 twice. For the upper bound, let $\Sigma_{1}=\lambda_{\min } I_{n}$ and $\Sigma_{2}=Q_{\hat{a}}$. Then $\Sigma_{2} \geq \Sigma_{1}$, and therefore according to theorem $2, P\left(\check{a}_{\mathrm{LSQ}}=a\right) \leq P\left(\check{a}_{\mathrm{LSQ}}^{\Sigma_{1}}=a\right)$. Since $\Sigma_{1}$ is diagonal, $\check{a}_{\mathrm{LSQ}}^{\Sigma_{1}}$ corresponds to a componentwise integer rounding of the entries of $\hat{a}^{\Sigma_{1}}$. This, combined with the independence of the entries of $\hat{a}^{\Sigma_{1}}$, gives $P\left(\check{a}_{\mathrm{LSQ}}^{\Sigma_{1}}=a\right)=\prod_{i=1}^{n} P\left(\left|\hat{a}_{i}^{\Sigma_{1}}-a_{i}\right| \leq \frac{1}{2}\right)$. Since the variances of $\hat{a}_{i}^{\Sigma_{1}}, i=1, \ldots, n$, are all the same and equal to $\lambda_{\min }$, we finally obtain $\prod_{i=1}^{n} P\left(\left|\hat{a}_{i}^{\Sigma_{1}}-a_{i}\right| \leq \frac{1}{2}\right)=$ $\left(2 \Phi\left(\frac{1}{2 \sqrt{\lambda_{\min }}}\right)-1\right)^{n}$. This concludes the proof of the upper bound. The proof of the lower bound follows in a similar way by choosing $\Sigma_{1}=Q_{\hat{a}}$ and $\Sigma_{2}=\lambda_{\max } I_{n}$.

The two bounds of Eq. (19) coincide when the two extreme eigenvalues coincide. This will be the case when the ambiguity VC matrix itself is a scaled unit matrix. In the actual practice of GPS this will not happen. How- ever, in order for the bounds to be sharp we would like the difference between the two eigenvalues to be small. This will usually not be the case when the VC matrix of the DD ambiguities is used. This shows that we should apply Eq. (19) to the VC matrix of the decorrelated ambiguities as produced by the LAMBDA method. By means of the decorrelation process of this method, the elongation of the ambiguity search space is considerably reduced. Hence, the above bounds are much sharper when using the eigenvalues of the transformed ambiguity $\mathrm{VC}$ matrix, than when using the eigenvalues of the original DD ambiguity VC matrix.

\section{References}

Anderson TW (1996) Some inequalities for symmetric convex sets with applications. Ann Statist 24(2): 753-762

Bock Y (1998) Medium distance GPS measurements. In: Teunissen PJG, Kleusberg A (eds) GPS for geodesy, 2nd edn. Springer, Berlin Heidelberg New York

Chmielewsky MA (1981) Elliptically symmetric distributions: a review and bibliography. Int Statist Rev 49: 67-74

de Jonge PJ, Tiberius CCJM (1996) The LAMBDA method for integer ambiguity estimation: implementation aspects. LGR Series, no. 12, Delft Geodetic Computing Centre, Delft University of Technology

Euler HJ, Goad C (1991) On optimal filtering of GPS dual freqeuncy observations without using orbit information. Bull Geod 65: $130-143$

Gerdan GP (1995) A comparison of four methods of weighting double difference pseudo range measurements. Trans Tasman Surv 1(1): 60-66

Gianniou M (1996) Genauigkeitssteigerung bei kurzzeit-statischen und kinematischen Satellitenmessungen bis hin zu Echtzeitanwendung. PhD thesis, Deutsche Geodaetische Kommission, Reihe C, no. 458, Muenchen

Hofmann-Wellenhof B, Lichtenegger H, Collins J (1997) Global positioning system: theory and practice, 4th edn. Springer, Berlin Heidelberg New York

Jin XX, de Jong CD (1996) Relationship between satellite elevation and precision of GPS code observations. J Navig 49: 253265

Jonkman NF (1997) Integer GPS-ambiguity estimation without the receiver-satellite geometry. MSc thesis, also published in LGR Series, no. 18 (1998) Delft Geodetic Computing Centre, Delft University of Technology

Leick A (1995) GPS satellite surveying, 2nd edn. John Wiley, New York

Parkinson B, Spilker JJ (eds) (1996) GPS: theory and applications, vols 1 and 2. AIAA, Washington, DC

Schaffrin B, Bock Y (1988) A unified scheme for processing GPS dual-band observations. Bull Geod 62: 142-160

Strang G, Borre K (1997) Linear algebra, geodesy, and GPS. Wellesley-Cambridge Press

Teunissen PJG (1993) Least-squares estimation of the integer GPS ambiguities. Invited lecture, Sect. IV Theory and Methodology, IAG General Meeting, Beijing, August. Also in: LGR Series, no. 6, Delft Geodetic Computing Centre, Delft University of Technology

Teunissen PJG (1995) The invertible GPS ambiguity transformations. Manuscr Geod 20: 489-497

Teunissen PJG (1998a) The ionosphere-weighted GPS baseline precision in canonical form. J Geod 72: 107-117

Teunissen PJG (1998b) On the integer normal distribution of the GPS ambiguities. Artific Sat 33(2): 49-64

Teunissen PJG (1998c) An optimality property of the integer leastsquares estimator. J Geod 73: 587-593 
Teunissen PJG, Kleusberg A (eds) (1998) GPS for geodesy, 2nd edn. Springer, Berlin Heidelberg New York

Teunissen PJG, Jonkman NF, Tiberius CCJM (1998) Weighting GPS dual frequency observations: bearing the cross of crosscorrelation. GPS Solutions, 2(2): 28-37

Tiberius CCJM (1998) Recursive data processing for kinematic GPS surveying. Publ Geodesy, no. 45, Netherlands Geodetic Commission, Delft 